\title{
Periodo reproductivo del bagre chihuil Bagre panamensis (Siluriformes: Ariidae) en el sureste del Golfo de California
}

Reproductive period of the chihuil sea catfish Bagre panamensis (Siluriformes: Ariidae) inhabiting the southeast Gulf of California

\section{Iram Zavala-Leal ${ }^{1,2^{*}}$, Deivis Palacios-Salgado ${ }^{1,2}$, Marcial Ruiz-Velazco ${ }^{1,2}$, José Trinidad $^{2}$ Nieto-Navarro ${ }^{1,2}$, Marco Antonio Cadena-Roa ${ }^{3}$, Delia Domínguez-Ojeda ${ }^{2}$, Juan Manuel Pacheco-Vega ${ }^{1,2}$ y Francisco Valdez-González ${ }^{2}$}

'Posgrado en Ciencias Biológico Agropecuarias, Universidad Autónoma de Nayarit, Km. 9 Carretera Tepic-Compostela, C.P. 63780, Xalisco, Nayarit, México

${ }^{2}$ Escuela Nacional de Ingeniería Pesquera, Universidad Autónoma de Nayarit, Km. 12 Carretera Los Cocos, C.P. 63740, San Blas, Nayarit, México

${ }^{3}$ Departamento de Pesquerías, Universidad Autónoma de Baja California Sur, Km. 5.5 Carretera al Sur, C.P. 23080, La Paz, Baja California Sur, México

*Autor de correspondencia: ziram28@hotmail.com

\begin{abstract}
Chihuil sea catfish Bagre panamensis is a fishing resource exploited throughout the year and without restrictions on the central coast of the Mexican Pacific. In order to properly manage the commercial fishery of some resource, it is important to know aspects of its biology such as reproduction. In the present study, the reproduction period was estimated through the gonadosomatic index (GSI), hepatosomatic index (HSI) and the condition factor of B. panamensis. Samples were obtained monthly between November 2015 and October 2016 of commercial fishing from San Blas area. A total of 346 specimens were collected, which 182 were females and 164 males. The sizes (total length and weight) found did not show differences between genders. Sex ratio was not different from 1:1 for the total of the samples. The weight-length relationship showed that both sexes presented a positive allometric growth type. According to the gonadosomatic (GSI) and hepatosomatic index (HSI), this specie has a breeding period per year, which runs from May to August. Based on these results, a management strategy for this fishery could be proposed in the southeastern Gulf of California.
\end{abstract}

Key words: Bagre panamensis, reproduction, weight-length relationships, sexual ratio

\begin{abstract}
Resumen.- El bagre marino Bagre panamensis es un recurso pesquero que se explota a lo largo de todo el año y sin restricción alguna en el sureste del Golfo de California. Para manejar de manera adecuada la pesquería comercial de algún recurso, es importante conocer aspectos de su biología básica como la reproducción. En el presente estudio se estimó el periodo de reproducción a través de los índices gonadosomático (IGS), hepatosomático (IHS) y el factor de condición de B. panamensis. Las muestras fueron obtenidas mensualmente entre noviembre de 2015 y octubre de 2016 de la pesca comercial del área de San Blas. Se recolectaron 346 ejemplares de los cuales 182 fueron hembras y 164 machos. Las tallas (longitud total y peso) encontradas no mostraron diferencias entre sexos. La proporción sexual no fue diferente de 1:1 para el total de las muestras. La relación peso-longitud mostró que ambos sexos presentaron un tipo de crecimiento alométrico positivo. De acuerdo al índice gonadosomático (IGS) y el hepatosomático (IHS) se observó que esta especie presenta un periodo de reproducción al año, que va de mayo a agosto. Con base en estos resultados, se podría proponer una estrategia de manejo para esta pesquería en el sureste del Golfo de California.
\end{abstract}

Palabras clave: Bagre panamensis, índice gonadosomático, índice hepatosomático, proporción sexual

\section{INTRODUCCIÓN}

Los bagres marinos o chihuiles pertenecen al orden Siluriformes. Este grupo de peces presenta una alta diversidad, principalmente en la región tropical y subtropical (Monasterio de Gonzo 2003). Los bagres que se distribuyen en ambientes marinos se limitan a las zonas costeras y suelen ser abundantes en aguas turbias, particularmente en estuarios y lagunas costeras de manglares (Acero 2002). Este grupo comprende 40 familias, 490 géneros y más de 3.730 especies (Nelson et al. 2016). Específicamente, la familia Ariidae está integrada por 147 especies a nivel global (Fricke 2018). En el Pacífico mexicano se reconoce la existencia de once especies, todas de importancia ecológica y algunas con importancia económica. El bagre marino, Bagre panamensis
(Gill, 1863) se caracteriza por presentar una coloración azul oscuro en el dorso, con lustre bronceado; plateado en los costados y blanco en el vientre, aletas oscuras a claras. Esta especie alcanza una talla de $57 \mathrm{~cm}$ de longitud total, habita comúnmente aguas marinas costeras de profundidades hasta $177 \mathrm{~m}$. Se distribuye desde el sur de California y Golfo de California hasta el norte de Perú (Robertson \& Allen 2008).

Dentro de la familia Ariidae son muy pocas las especies en México que reportan aspectos sobre su reproducción. En el Golfo de México, la especie más estudiada ha sido el bagre bandera Bagre marinus (Mitchill, 1815), de la que se ha descrito en el sureste de Campeche un periodo de reproducción de abril a octubre (Caballero-Chávez 2013), mientras que en las costas de Tabasco es entre junio 
y agosto (Segura-Berttolini \& Mendoza-Carranza 2013). En el Pacífico mexicano, la especie más estudiada es el bagre cominate Occidentarius platypogon Günther, 1864. En estudios sobre su reproducción se ha reportado que el periodo de reproducción es de mayo a agosto (Amezcua \& Muro-Torres 2012). Otra especie de la que se han descrito aspectos reproductivos es Galeichthys caerulescens (actualmente Ariopsis guatemalensis) (Yáñez-Arancibia et al. 1976). Es probable que esta especie se reproduzca con una intensidad bimensual debido a que se han encontrado machos incubando huevos y protegiendo larvas en sus bocas, principalmente en septiembre, octubre y mayo. Para B. panamensis, Muro \& Amezcua (2011) reportaron que en la bahía de Mazatlán presenta un periodo de reproducción entre mayo y agosto.

En San Blas, Nayarit, en el sureste del Golfo de California existen diversas especies de bagres que son explotadas a nivel comercial. De acuerdo con los pescadores de la cooperativa, una de las más capturadas es B. panamensis, localmente conocido como bandera. De acuerdo con el anuario estadístico de acuacultura y pesca, en el 2014 la producción total del estado fue de $1.859 \mathrm{t}$ de bagres marinos, que corresponde al $18 \%$ de la producción total nacional (SAGARPA 2015). En esta zona, el bagre bandera Bagre panamensis es una especie de importancia comercial, y es un recurso pesquero que se explota todo el año y sin regulación alguna. Para manejar de manera adecuada la pesquería comercial de algún recurso, es importante determinar su biología básica. Es por ello, que en el presente trabajo se estima el periodo de reproducción de B. panamensis, que contribuya en conjunto con otros estudios a establecer un manejo pesquero de esta especie en la región.

\section{Materiales Y MÉTOdos}

Se realizaron muestreos mensuales entre noviembre 2015 y octubre 2016. Los organismos fueron obtenidos de la pesca comercial ribereña que opera en el área de San Blas entre los $21,41^{\circ} \mathrm{N}-05,20^{\circ} \mathrm{W}$ y los $21,55^{\circ} \mathrm{N}-105,37^{\circ} \mathrm{W}$, con diferentes sistemas de pesca como palangres, redes de enmalle ( 3 a 4,5 ") y redes de arrastre camaroneras (2,5 a 3"). De todos los organismos obtenidos se registró la longitud total (Lt) y el peso total (P). Posteriormente, se realizó una incisión abdominal y se extrajeron las gónadas y el hígado, las cuales se pesaron de manera individual. El sexo de cada organismo se registró a través de la observación directa de las gónadas.

La talla entre sexos se comparó a través de la prueba no paramétrica de Kruskal-Wallis. Se estimó la proporción sexual para cada muestreo, así como para el total de muestras obtenidas. Para determinar si la proporción sexual difiere de 1:1, se realizó una prueba de chi-cuadrado $\left(\chi^{2}\right)$ (Zar 2010). Se estimó la relación peso-longitud (RPL) por sexos; para determinar si hubo diferencias entre sexos se realizó un análisis de covarianzas (ANCOVA). El tipo de crecimiento se obtuvo a través de la comparación de la pendiente (b) con el valor hipotético de isometría (3), mediante la prueba t de Student (Zar 2010).

Además, se estimaron los índices morfofisiológicos para determinar el periodo reproductivo. El índice gonadosomático (IGS) se calculó mediante la ecuación IGS= peso de la gónada/peso del pez sin gónada $\mathrm{x} 100(\mathrm{De}$ Vlaming et al. 1982), el índice hepatosomático (IHS) por la ecuación IHS = peso del hígado/peso del pez sin hígado x 100 (Rodríguez-Gutiérrez 1992), y el factor de condición por $\mathrm{K}=$ Peso del pez sin gónada e hígado/longitud total del pez elevado al valor de $b$, es decir, la pendiente de la RPL (Nikolsky 1963). Las variaciones mensuales se analizaron mediante la prueba no paramétrica de Kruskal-Wallis debido a que los datos no cumplían los supuestos para realizar una prueba paramétrica (Zar 2010).

\section{Resultados}

Se obtuvieron un total de 346 ejemplares de B. panamensis, de los cuales 182 fueron hembras y 164 machos. Las tallas máximas y mínimas registradas para hembras fueron de 47 cm de Lt y $990 \mathrm{~g}$ de peso y 20,6 cm y $148 \mathrm{~g}$, respectivamente, mientras que para los machos fueron de $41,5 \mathrm{~cm}$ de Lt y 688 $\mathrm{g}$ de peso y $22,2 \mathrm{~cm}$ y $65 \mathrm{~g}$, respectivamente. Sin embargo, no se observaron diferencias significativas $(\mathrm{H}=0,53, P=$ 0,82 ) en cuanto a la longitud total entre hembras (promedio \pm desviación estándar, $34,4 \pm 4,7)$ y machos $(33,1 \pm 4,4)$ (Fig. 1), ni en cuanto al peso de los organismos $(\mathrm{H}=0,21, P$ $=0,64)(360,4 \pm 164,5$ y $317,4 \pm 139,2$, hembras y machos, respectivamente).

En cuanto a la proporción sexual del total de las muestras (1:1,1 M:H), se observó que no fue diferente de $1: 1$, excepto en marzo (1:0,3 M:H) y octubre (1:2,5 M:H) (Tabla 1). De acuerdo a la relación peso-longitud, no se observaron diferencias significativas entre sexos (ANCOVA, $F=0,42$, $P=0,52)$, por lo tanto se estimó la relación para sexos combinados. Se observó que B. panamensis presentó un crecimiento de tipo alométrico positivo, registrando un valor de la pendiente $(b=3,28)$ estadísticamente mayor al valor hipotético de isometría que es igual a tres $(\mathrm{t}=1,96, P$ $=0,0001$ ) (Fig. 2). 


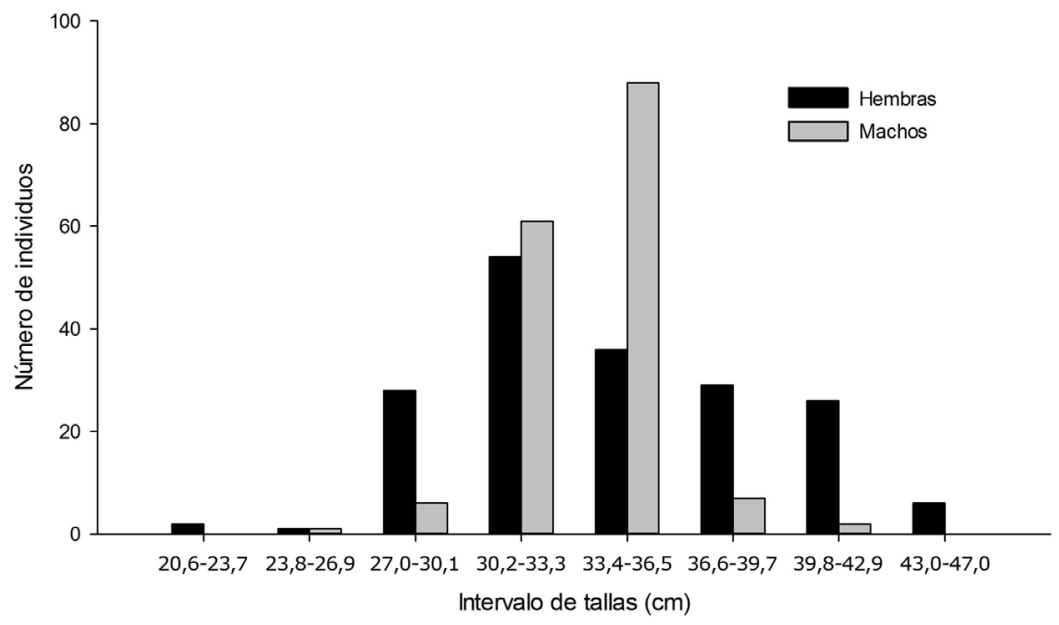

Figura 1. Histograma de frecuencia de tallas de Bagre panamensis en el sureste del Golfo de California / Size frequency histogram of Bagre panamensis from the southeast Gulf of California

Tabla 1.- Proporción sexual de Bagre panamensis en el sureste del Golfo de California durante 2015-2016 / Sex ratio of Bagre panamensis on the southeast Gulf of California during 2015-2016

\begin{tabular}{cccccc}
\hline Meses & M & H & $\begin{array}{c}\text { Proporción } \\
\text { M:H }\end{array}$ & $\chi^{2}$ & $P$ \\
\hline Nov & 14 & 16 & $1: 1,14$ & 0,133 & 0,7153 \\
Dic & 14 & 6 & $1: 0,43$ & 3,200 & 0,0736 \\
Ene & 16 & 23 & $1: 1,44$ & 1,256 & 0,2624 \\
Feb & 20 & 17 & $1: 0,85$ & 0,243 & 0,6220 \\
Mar & 21 & 8 & $1: 0,38$ & 5,828 & $0,0157^{*}$ \\
Abr & 15 & 10 & $1: 0,67$ & 1,000 & 0,3173 \\
May & 11 & 20 & $1: 1,82$ & 2,613 & 0,1059 \\
Jun & 16 & 19 & $1: 1,19$ & 0,257 & 0,6121 \\
Jul & 2 & 7 & $1: 3,50$ & 2,778 & 0,0955 \\
Ago & 13 & 16 & $1: 1,23$ & 0,310 & 0,0678 \\
Sep & 13 & 17 & $1: 1,31$ & 0,533 & 0,4653 \\
Oct & 9 & 23 & $1: 2,56$ & 6,125 & $0,0133^{*}$ \\
Total & 164 & 182 & $1: 1,11$ & 0,936 & 0,3333
\end{tabular}

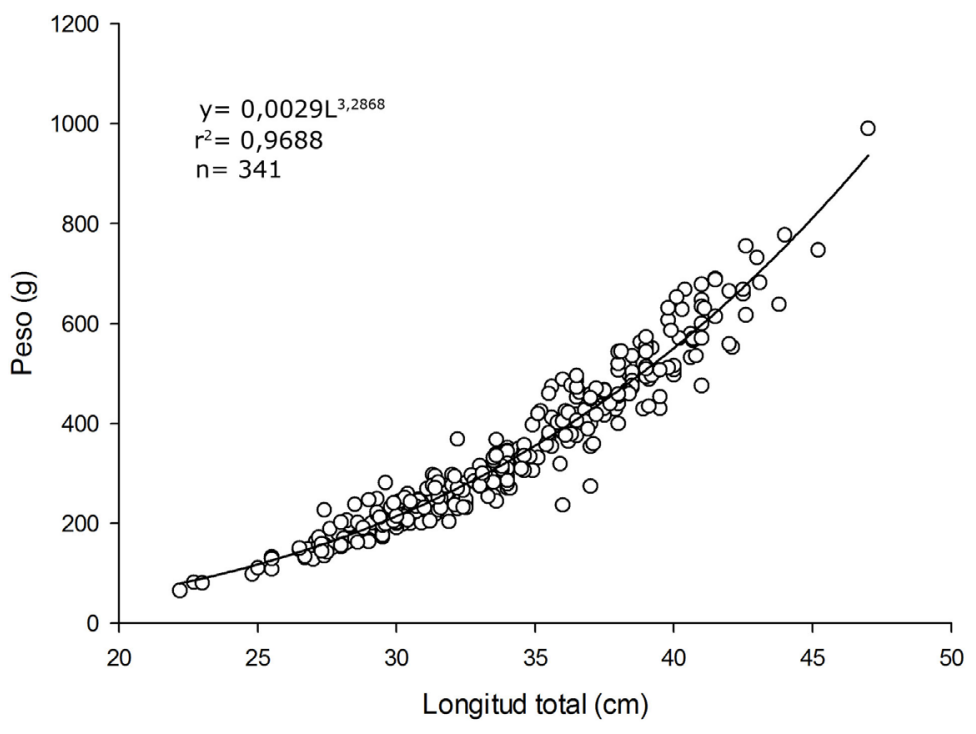

El IGS mostró un pico durante febrero (media $=0,46$ en hembras y 0,21 en machos) y otro entre mayo $(1,37)$ y agosto $(3,88)$ en hembras alcanzando el pico máximo en agosto (Fig. 3). En machos, el segundo pico fue de mayo a junio $(0,10$ y 0,17 , respectivamente) (Fig. 3). El IHS no mostró un patrón claro con respecto al evento reproductivo, sin embargo, se observó un incremento en su valor en los meses de mayor actividad reproductiva (Fig. 4). En hembras se observó que dentro de los meses con valores más altos se presentaron en mayo $(1,88)$, agosto $(1,68)$ y febrero $(1,45)$, en los cuales también se presentaron los valores más altos del IGS (Fig. 4). En machos, se observó un patrón de comportamiento de este índice muy similar al de hembras (Fig. 4). De acuerdo con el IGS y el IHS, se observó que el bagre presenta un periodo reproductivo que abarca de mayo a agosto. No obstante, los resultados de estos índices sugieren que podría presentarse otro pequeño pico reproductivo en febrero, aunque hasta el momento no existe suficiente evidencia para considerarlo como un evento
Figura 2. Relación peso-longitud de Bagre panamensis en el sureste del Golfo de California / Length-weight relationships of Bagre panamensis from the southeast Gulf of California 


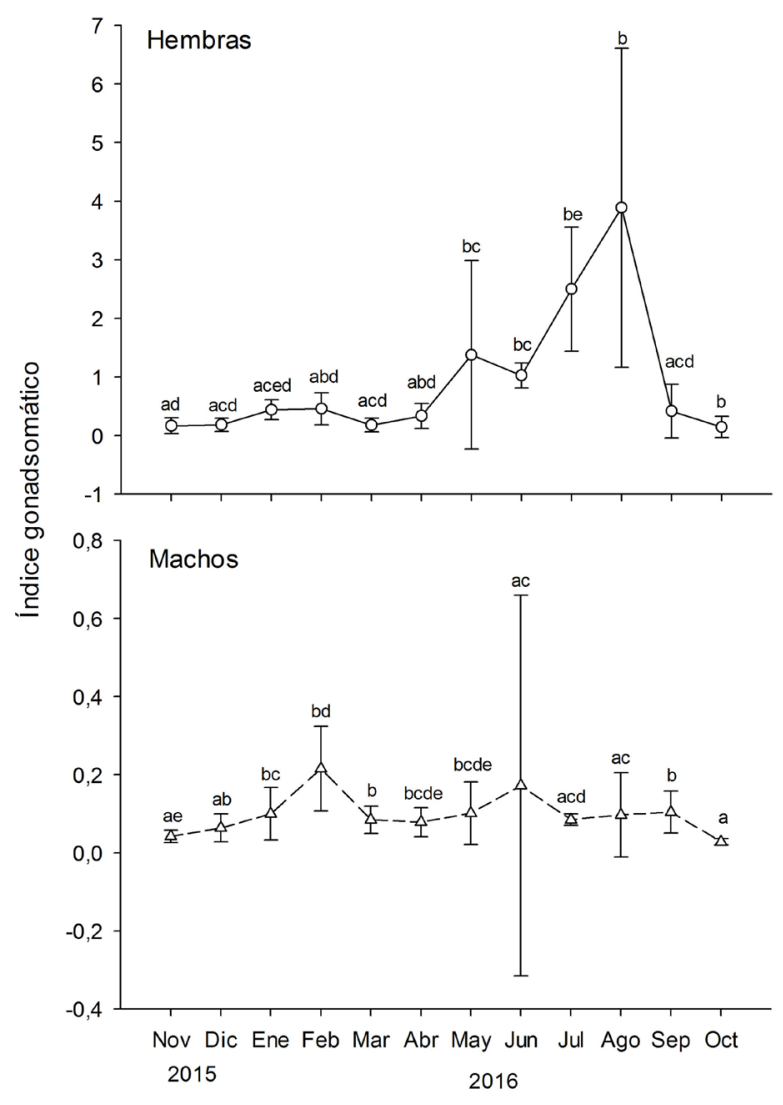

Figura 3. Variación mensual del índice gonadosomático de Bagre panamensis. Los valores corresponden a los promedios y las barras a la desviación estándar. Letras diferentes indican diferencias significativas $(P<\mathbf{0 , 0 5}) /$ Monthly variation of the gonadosomatic index of Bagre panamensis. The values correspond to the means and the bars to the standard deviation. Different letters indicate significant differences $(P$ $<0.05)$

reproductivo. En cuanto al factor de condición relativo (K), se observó un patrón similar entre ambos sexos (Fig. 5). Se observaron dos picos, uno en enero y otro en junio para ambos sexos, sin embargo, en machos se observó un tercer pico en octubre. Para hembras el valor de K en enero fue de 0,56 y en junio de 0,62 (Fig. 5); mientras que en machos los valores de $\mathrm{K}$ en enero y junio fueron de $0,23 \mathrm{y}$ en octubre fue de 0,24 (Fig. 5).

\section{DisCUSIÓN}

El bagre marino, B. panamensis, es una especie de importancia comercial en la región de Nayarit. Las tallas de los organismos analizados fueron de 20,6 a $47 \mathrm{~cm}$ de Lt y de 65 a 990 g de peso, los cuales provenían de la pesca comercial ribereña. Las tallas encontradas de hembras y machos durante este estudio no mostraron diferencias

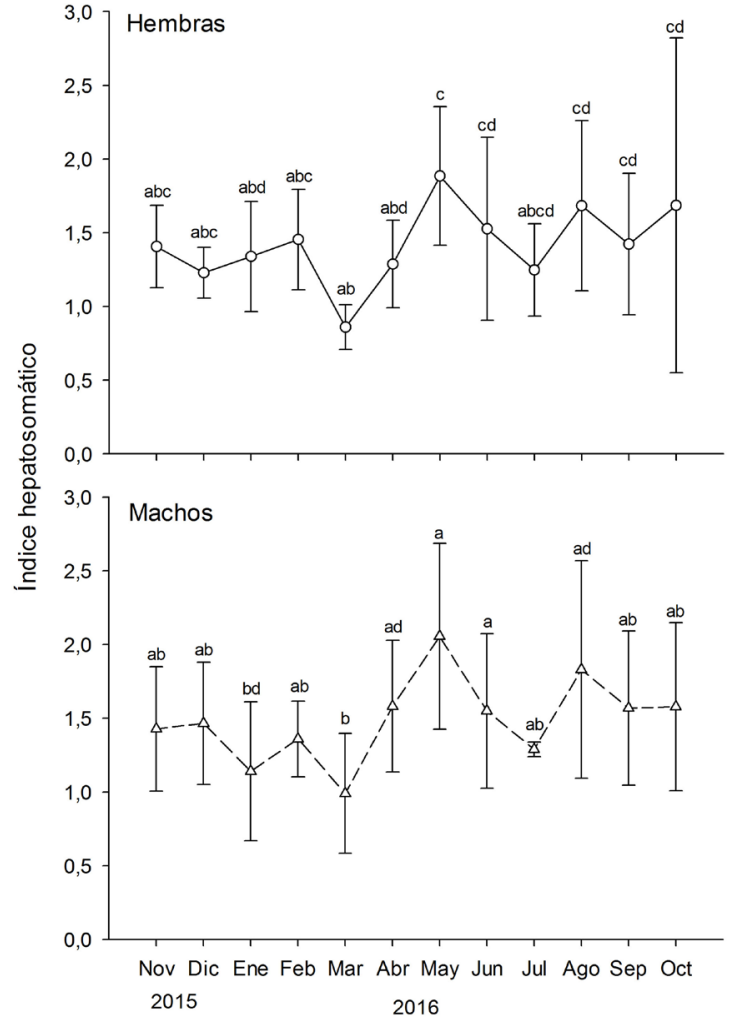

Figura 4. Variación mensual del índice hepatosomático de Bagre panamensis. Los valores corresponden a los promedios y las barras a la desviación estándar. Letras diferentes indican diferencias significativas $(P<0,05) /$ Monthly variation of the hepatosomatic index of Bagre panamensis. The values correspond to the means and the bars to the standard deviation. Different letters indicate significant differences $(P<0.05)$

significativas, como se ha reportado para B. marinus en Brasil (Pinheiro et al. 2006) y en México (Caballero-Chávez 2013) mostrando que no existe un dimorfismo sexual referido a la talla como ha sido reportado para otros aríidos (Rimmer \& Merrick 1983), sin embargo, esto también puede estar en función de las tallas capturadas (selectividad del arte de pesca). De manera general, se observó una dominancia de hembras (182) sobre los machos (164) tal y como se ha reportado para otras especies de la familia Ariidae (Pinheiro et al. 2006, Queiroga et al. 2012). La proporción sexual fue diferente de $1: 1$ en marzo $(1: 0,85, \mathrm{M}: \mathrm{H})$ y en octubre $(1: 2,56)$; sin embargo, no hubo diferencia para el total de las muestras (1:1,11, M:H). Este fenómeno ha sido reportado para otras especies de bagres (Lara-Domínguez et al. 1981, Queiroga et al.2012, Caballero-Chávez 2013). Sin embargo, en $B$. marinus del Noreste de Brasil la proporción sexual 


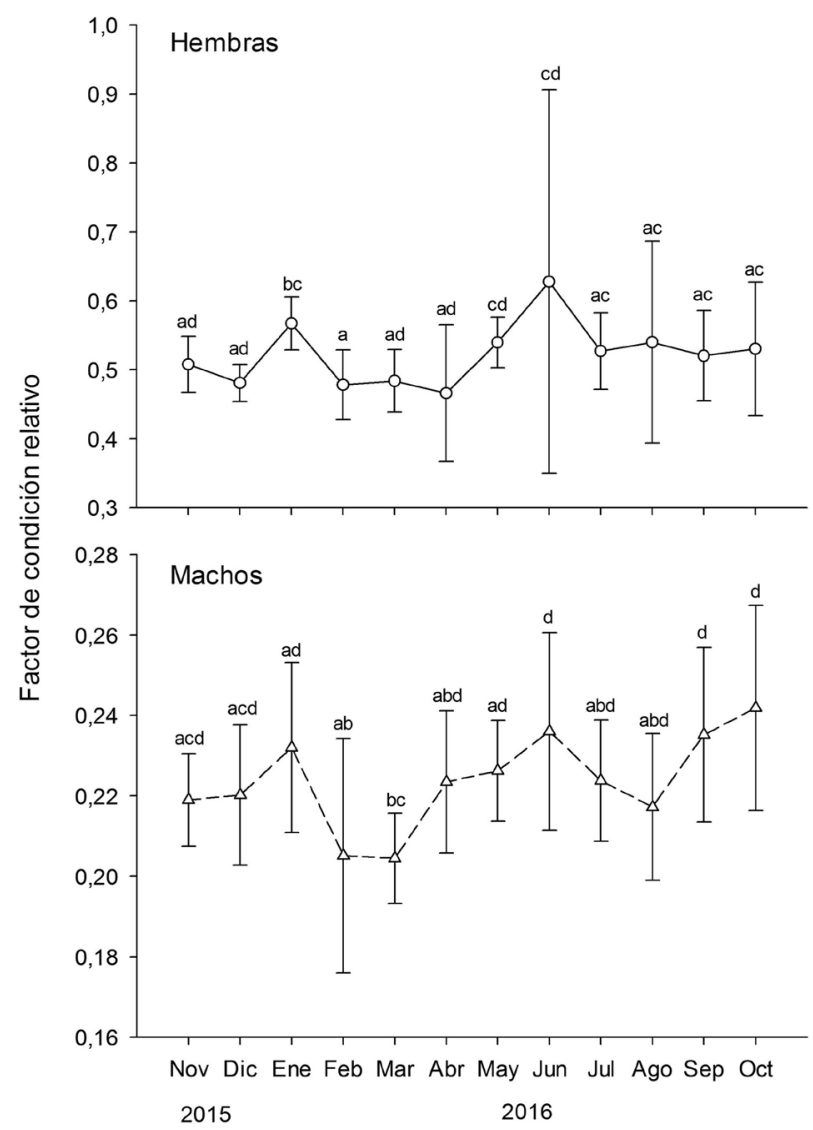

Figura 5. Variación mensual del factor de condición relativo de Bagre panamensis. Los valores corresponden a los promedios y las barras a la desviación estándar. Letras diferentes indican diferencias significativas $(P<0,05)$ / Monthly variation of the relative condition factor of Bagre panamensis. The values correspond to the means and the bars to the standard deviation. Different letters indicate significant differences $(P<0.05)$

total fue de 1:2,52 (M:H) (Pinheiro et al. 2006). De acuerdo con Nikolsky (1963) y Gómez et al. (1994), la proporción sexual 1:1 es la más esperada para la mayoría de las especies de peces, y es más común encontrar esta proporción cuando se evalúa el total de las muestras, aunque en algunos casos se encuentran diferencias en las evaluaciones mensuales debido a distintos comportamientos entre sexos (Vazzoler 1996). La relación peso-longitud mostró que $B$. panamensis presenta un crecimiento alométrico positivo $(\mathrm{b}>3)$, es decir, existe una mayor ganancia proporcional en peso que en longitud, lo cual es común en peces (Wootton 1990, Froese 2006). Esto mismo ha sido reportado para B. marinus del Golfo de México (Mendoza-Carranza \& Hernández-Franyutti 2005). En especies como Arius melanopus (actualmente Cathorops melanopus) se ha observado un crecimiento alométrico positivo y un crecimiento alométrico negativo (Lara-Domínguez et al. 1981). El tipo de crecimiento puede estar en función de cambios ambientales, la alimentación, el metabolismo individual, la madurez sexual, entre otros (Candia et al. 1973). Además de factores biológicos y ambientales, el tipo de crecimiento observado en una población puede estar influenciado por la forma de muestreo realizada. Se puede introducir un sesgo en el muestreo si solo se capturan organismos de tallas similares, por ejemplo, si se utiliza un solo tipo de sistema de pesca (redes de enmalle de una sola luz de malla, palangres de un solo tamaño de anzuelo, etc.) (Froese 2006).

Los índices morfofisiológicos como IGS, IHS y el factor de condición, se utilizan con frecuencia para evaluar el periodo reproductivo en peces (Kanak \& Tachihara 2008, González-Castro \& Minos 2016). En este estudio, se observó a través del IGS e IHS que existe un periodo de reproducción desde mayo a agosto (final de la primavera y verano) y se observaron indicios de otro probable periodo de reproducción en febrero (final del invierno). Muro \& Amezcua (2011) reportaron (a través de una escala morfocromática e índices morfofisiológicos) que B. panamensis en la bahía de Mazatlán presenta un solo periodo reproductivo al año, que va de mayo a agosto, que coincide con el periodo encontrado en nuestro estudio. De acuerdo con esto, se sugiere emplear una técnica más precisa para la evaluación de las gónadas de los bagres de la costa de San Blas, Nayarit, para descartar o confirmar los resultados proporcionados por los índices morfo-fisiológicos. Dentro de las técnicas más precisas, para estas evaluaciones, se ha considerado la 
técnica histológica por lo detallado de la información que puede proporcionar (West 1990). En otras especies de bagres se ha reportado que existe un solo periodo de reproducción al año. Esto fue determinado a través de observaciones macroscópicas y microscópicas de las gónadas e índices morfofisiológicos para Sciadeichthys luniscutis (Gomes \& Araujo 2004), Genidens genidens (Gomes et al. 1999, Gomes \& Araujo 2004), Cathorops spixii (Gomes et al. 1999), Bagre marinus (Pinheiro et al. 2006), Occidentarius platypogon (Amezcua \& Muro-Torres 2012), Sciades guatemalensis (Yánez-Arancibia et al. 1976) y Bagre marinus (Caballero-Chávez 2013). Para B. marinus, también se ha determinado su periodo reproductivo únicamente mediante el uso de índices morfofisiológicos (MendozaCarranza \& Hernández-Franyutti 2005). En especies como Arius argyropleuron se ha reportado (empleando una escala morfo-cromática) que sexualmente madura durante todo el año con dos picos (abril y julio) para hembras y un pico (noviembre) para machos (Mansor et al. 2012). La época de reproducción en la mayoría de los bagres se presenta entre la primavera e inicio del otoño, que coincide con el periodo cálido del año o durante el aumento de la temperatura del agua (Dmitrenko 1970, Yañez-Arancibia et al. 1976, Rimmer \& Merrick 1983, Mendoza-Carranza \& Hernández-Franyutti 2005). Esta coincide con la temporada de precipitaciones y baja salinidad (Gomes et al. 1999, Mendoza-Carranza \& Hernández-Franyutti 2005). Estas condiciones prevalecen en la costa de San Blas, Nayarit, durante lo que puede ser el periodo de reproducción más importante de $B$. panamensis. Un factor importante que determina que el periodo de reproducción del bagre bandera sea de mayo a agosto podría ser el aporte de nutrientes proveniente de los ríos en la temporada de lluvias, lo cual ocurre en esos meses en la zona de estudio. Debido a que dichos aportes de ríos son una vía de fertilización para los mares (García-Hoyos et al. 2010), facilita la disponibilidad de alimento y con ello que se desencadenen los procesos para que ocurra el evento reproductivo. De igual forma, se ha reportado que es probable que el desove en bagres ocurra en época de lluvias para favorecer el desarrollo de los juveniles, puesto que les permite aprovechar las condiciones de los estuarios como áreas de crianza (Blaber et al. 1995).

Los resultados de este trabajo demuestran que $B$. panamensis presenta un periodo de reproducción de mayo a agosto en la costa de San Blas, Nayarit, de igual forma como se ha reportado para esta misma especie en bahía de Mazatlán. Esta información será útil para proponer estrategias de manejo adecuadas para esta especie en el sureste del Golfo de California.

\section{Agradecimientos}

Este trabajo fue realizado en el marco de la Red Interinstitucional para la Evaluación, Manejo y Aprovechamiento de los Recursos Acuáticos, a través del proyecto "Línea base para el desarrollo de planes de manejo pesquero y acuícola del bagre chihuil, Bagre panamensis (Siluriformes: Ariidae)" financiado por la Secretaria de Educación Pública (SEP-PRODEP). Los autores agradecen a los pescadores y comercializadores de pescado del puerto de San Blas, Nayarit por las facilidades para obtener las muestras biológicas. Agradecemos también a los estudiantes de la Escuela Nacional de Ingeniería Pesquera por el apoyo durante los muestreos. Finalmente, agradecemos a los tres revisores anónimos por sus valiosas aportaciones que enriquecieron este manuscrito.

\section{LITERATURA CITADA}

Acero PA. 2002. Family Ariidae. In: Carpenter KE (ed). The living marine resources of the western central Atlantic Vol. 2: 831-852. FAO, Roma.

Amezcua F \& V Muro-Torres. 2012. Biología reproductiva del bagre cominate Occidentarius platypogon (Pisces: Ariidae) en el sureste del golfo de California. Latin American Journal of Aquatic Research 40(2): 428-434.

Blaber SJM, DT Brewer \& JP Salini. 1995. Fish communities and the nursery role of the shallow inshore waters of a tropical bay in the Gulf of Carpentaria, Australia. Estuarine, Coastal and Shelf Science 40: 177-193.

Caballero-Chávez V. 2013. Madurez y reproducción de bagre bandera Bagre marinus en el sudeste de Campeche. Ciencia Pesquera 21(2): 13-19.

Candia CR, ML Baiz \& SE Cabrera. 1973. Algunos aspectos biológicos de las especies de ictiofauna de la zona de Punta Lara (Río de la Plata). III. Estudio de la edad y crecimiento del bagre porteño (Parapimelodus valenciennesi) con algunos datos sobre su reproducción, 33 pp. Servicio de Hidrografía Naval, Rio de La Plata.

De Vlaming V, G Grossman \& F Chapman. 1982. On the use of the gonadosomatic index. Comparative Biochemical and Physiology 73A(1): 31-39.

Fricke R, WN Eschmeyer \& R van der Laan. 2018. Catalog of fishes: genera, species, references. California Academy of Sciences. $<$ http://researcharchive.calacademy.org/research/ ichthyology/catalog/fishcatmain.asp $>$

Froese R. 2006. Cube law, condition factor and weight-length relationships: history, meta-analysis and recommendations. Journal of Applied Ichthyology 22: 241-253.

García-Hoyos LM, A Franco-Herrera, JS Ramírez-Barón \& DA López-Cerón. 2010. Dinámica océano-atmósfera y su influencia en la biomasa fitoplanctónica, en la zona costera del departamento del Magdalena, Caribe colombiano. Boletín de Investigaciones Marinas y Costeras 39(2): $307-$ 335.

Gomes ID \& FG Araujo. 2004. Reproductive biology of two marine catfishes (Siluriformes, Ariidae) in the Sepetiba Bay, Brazil. Revista de Biología Tropical 52(1): 143-156. 
Gomes ID, FG Araújo, MC Costa de Azevedo \& AL Machado-Pessanha. 1999. Biologia reprodutiva dos bagres marinos Genidens genidens (Valenciennes) e Cathorops spixii (Agassiz) (Siluriformes, Ariidae), na Baía de Sepetiba, Rio de Janeiro, Brasil. Revista Brasileira de Zoologia 16(2): 171-180.

Gómez G, R Guzmán \& L Marcano. 1994. Aspectos biométricos y de reproducción del pargo ojo amarillo Lutjanus vivanus (Cuvier, 1828) de las islas Los Hermanos, Venezuela. Zootecnia Tropical 12(2): 259-280.

González-Castro M \& G Minos. 2016. Sexuality and reproduction of Mugilidae. In: Crosetti D \& S Blaber (eds). Biology, ecology and culture of grey mullets (Mugilidae), pp. 227-263. CRC Press, Boca Raton.

Kanak MK \& K Tachihara. 2008. Reproductive biology of common silver biddy Gerres oyena in Okinawa Island of southern Japan. Fisheries Science 74: 265-275.

Lara-Domínguez AL, A Yáñez-Arancibia \& F AmézcuaLinares. 1981. Biología y ecología del bagre Arius melanopus Günther en la laguna de términos, sur del Golfo de México (Pisces: Ariidae). Anales del Centro del Mar y Limnología, Universidad Nacional Autónoma, México 8(1): 267-304.

Mansor MI, MN Nurul-Shafikah, Y Khairun \& MN SitiAzizah. 2012. Reproductive biology of estuarine catfish, Arius argyropleuron (Siluriformes: Ariidae) in the northern part of Peninsular Malaysia. Journal of Biology, Agriculture and Healthcare 2(3): 14-28.

Mendoza-Carranza M \& A Hernández-Franyutti. 2005. Annual reproductive cycle of gafftopsail catfish, Bagre marinus (Ariidae) in a tropical coastal environment in the Gulf of Mexico. Hidrobiológica 15(3): 275-282.

Monasterio de Gonzo G. 2003. Peces de los ríos Bermejo, Juramento y Cuencas Endorreicas de la Provincia de Salta, 243 pp. Museo de Ciencias Naturales y Consejo de Investigación, Universidad Nacional de Salta, Salta.

Muro V \& F Amezcua. 2011. Observation on the reproductive biology of the chihuil sea catfish in the southeast Gulf of California: Implications for management. American Fisheries Society 77: 1-9.

Nelson JS, TC Grande \& MVH Wilson. 2016. Fishes of the world, 752 pp. John Wiley and Sons, Hoboken.

Nikolsky GV. 1963. The ecology of fishes, $352 \mathrm{pp}$. Academic Press, New York.
Pinheiro P, MK Broadhurst, FHV Hazin, T Bezerra \& S Hamilton. 2006. Reproduction in Bagre marinus (Ariidae) off Pernambuco, northeastern Brazil. Journal of Applied Ichthyology 22: 189-192.

Queiroga FR, JE Golzio, RB dos Santos, TO Martins \& AL Vendel. 2012. Reproductive biology of Sciades herzbergii (Siluriformes: Ariidae) in a tropical estuary in Brazil. Zoologia 29(5): 397-404.

Rimmer MA \& JR Merrick. 1983. A review of reproduction and development in the fork-tailed catfishes (Ariidae). Proceedings of the Linnean Society of New South Wales 107: $41-50$

Robertson DR \& GR Allen. 2008. Shore fishes of the Tropical Eastern Pacific: an information system on line. Ver 1.0 (2008). Smithsonian Tropical Research Institute, Balboa. $<$ www.neotropicalfishes.org/sftep >

Rodríguez-Gutiérrez M. 1992. Técnicas de evaluación cuantitativa de la madurez gonádica en peces, 79 pp. AGT Editor, México.

SAGARPA. 2015. Anuario estadístico de acuacultura y pesca 2013. Secretaria de Agricultura, Ganadería, Desarrollo Rural, Pesca y Alimentación, México. <https://www.gob.mx/ conapesca/documentos/anuario-estadistico-de-acuaculturay-pesca>

Segura-Berttolini EC \& M Mendoza-Carranza. 2013. La importancia de los machos del bagre bandera, Bagre marinus (Pisces: Ariidae), en el proceso reproductivo. Ciencias Marinas 39(1): 29-39.

Vazzoler AEAM. 1996. Biologia da reprodução de peixes teleósteos: teoria e prática, 169 pp. Maringá, EDUEM, Sao Paulo.

Yáñez-Arancibia A, J Curiel-Gómez \& VL de Yáñez. 1976. Prospección biológica y ecológica del bagre marino Galeichthys caerulescens (Gunther) en el sistema lagunar costero de Guerrero, México (Pisces: Ariidae). Anales del Centro del Mar y Limnología, Universidad Nacional Autónoma de México 3: 125-138.

West G. 1990. Methods of assessing ovarian development in fishes: A review. Australian Journal of Marine and Freshwater Research 41: 199-222.

Wootton RJ. 1990. Ecology of teleost fishes, 386 pp. Chapman \& Hall, London.

Zar JH. 2010. Biostatistical analysis, 944 pp. Prentice Hall, Upper Saddle River. 